\title{
Improved Constraints on Primordial Gravitational Waves using Planck, WMAP, and BICEP/Keck Observations through the 2018 Observing Season
}

P. A. R. Ade, ${ }^{1}$ Z. Ahmed, ${ }^{2}$ M. Amiri, ${ }^{3}$ D. Barkats, ${ }^{4}$ R. Basu Thakur, ${ }^{5}$ C. A. Bischoff, ${ }^{6}$ D. Beck, ${ }^{2,7}$ J. J. Bock, ${ }^{5,8}$ H. Boenish, ${ }^{4}$ E. Bullock, ${ }^{9}$ V. Buza, ${ }^{10}$ J. R. Cheshire IV, ${ }^{9}$ J. Connors, ${ }^{4}$ J. Cornelison, ${ }^{4}$ M. Crumrine, ${ }^{11}$ A. Cukierman, ${ }^{7,2}$ E. V. Denison, ${ }^{12}$ M. Dierickx, ${ }^{4}$ L. Duband, ${ }^{13}$ M. Eiben,${ }^{4}$ S. Fatigoni, ${ }^{3}$ J. P. Filippini, ${ }^{14,15}$ S. Fliescher, ${ }^{11}$ N. Goeckner-Wald, ${ }^{7}$ D. C. Goldfinger, ${ }^{4}$ J. Grayson, ${ }^{7}$ P. Grimes, ${ }^{4}$ G. Hall, ${ }^{11}$ G. Halal, ${ }^{7}$ M. Halpern, ${ }^{3}$ E. Hand, ${ }^{6}$ S. Harrison, ${ }^{4}$ S. Henderson, ${ }^{2}$ S. R. Hildebrandt, ${ }^{5,8}$ G. C. Hilton, ${ }^{12}$ J. Hubmayr, ${ }^{12}$ H. Hui, ${ }^{5}$ K. D. Irwin, ${ }^{7,2,12}$ J. Kang, ${ }^{7,5}$ K. S. Karkare, ${ }^{4,10}$ E. Karpel, ${ }^{7}$ S. Kefeli, ${ }^{5}$ S. A. Kernasovskiy, ${ }^{7}$ J. M. Kovac, ${ }^{4,16}$ C. L. Kuo, ${ }^{7,2}$ K. Lau, ${ }^{11}$ E. M. Leitch, ${ }^{10}$ A. Lennox, ${ }^{14}$ K. G. Megerian, ${ }^{8}$ L. Minutolo, ${ }^{5}$ L. Moncelsi, ${ }^{5}$ Y. Nakato, ${ }^{7}$ T. Namikawa, ${ }^{17}$ H. T. Nguyen, ${ }^{8}$ R. O’Brient, ${ }^{5,8}$ R. W. Ogburn IV,${ }^{7,2}$ S. Palladino, ${ }^{6}$ T. Prouve ${ }^{13}$ C. Pryke,${ }^{11,9,}$ B. Racine, ${ }^{4,18}$ C. D. Reintsema ${ }^{12}$ S. Richter, ${ }^{4}$ A. Schillaci, ${ }^{5}$ R. Schwarz, ${ }^{11}$

B. L. Schmitt, ${ }^{4}$ C. D. Sheehy, ${ }^{19}$ A. Soliman, ${ }^{5}$ T. St. Germaine, ${ }^{4,16}$ B. Steinbach, ${ }^{5}$ R. V. Sudiwala, ${ }^{1}$ G. P. Teply, ${ }^{5}$ K. L. Thompson, ${ }^{7,2}$ J. E. Tolan, ${ }^{7}$ C. Tucker, ${ }^{1}$ A. D. Turner, ${ }^{8}$ C. Umiltà, ${ }^{6,14}$ C. Vergèss, ${ }^{4}$ A. G. Vieregg, ${ }^{20,10}$ A. Wandui, ${ }^{5}$ A. C. Weber, ${ }^{8}$ D. V. Wiebe, ${ }^{3}$ J. Willmert, ${ }^{11}$ C. L. Wong, ${ }^{4,16}$ W. L. K. Wu, ${ }^{2}$ H. Yang, ${ }^{7}$ K. W. Yoon, ${ }^{7,2}$ E. Young, ${ }^{7,2}$ C. Yu, ${ }^{7}$ L. Zeng, ${ }^{4}$ C. Zhang, ${ }^{5}$ and S. Zhang ${ }^{5}$

\section{(BICEP/Keck Collaboration)}

\author{
${ }^{1}$ School of Physics and Astronomy, Cardiff University, Cardiff CF24 3AA, United Kingdom \\ ${ }^{2}$ Kavli Institute for Particle Astrophysics and Cosmology, SLAC National Accelerator Laboratory, \\ 2575 Sand Hill Rd, Menlo Park, California 94025, USA \\ ${ }^{3}$ Department of Physics and Astronomy, University of British Columbia, Vancouver, British Columbia V6T 1Z1, Canada \\ ${ }^{4}$ Center for Astrophysics, Harvard \& Smithsonian, Cambridge, Massachusetts 02138, USA \\ ${ }^{5}$ Department of Physics, California Institute of Technology, Pasadena, California 91125, USA \\ ${ }^{6}$ Department of Physics, University of Cincinnati, Cincinnati, Ohio 45221, USA \\ ${ }^{7}$ Department of Physics, Stanford University, Stanford, California 94305, USA \\ ${ }^{8}$ Jet Propulsion Laboratory, Pasadena, California 91109, USA \\ ${ }^{9}$ Minnesota Institute for Astrophysics, University of Minnesota, Minneapolis, Minnesota 55455, USA \\ ${ }^{10}$ Kavli Institute for Cosmological Physics, University of Chicago, Chicago, Illinois 60637, USA \\ ${ }^{11}$ School of Physics and Astronomy, University of Minnesota, Minneapolis, Minnesota 55455, USA \\ ${ }^{12}$ National Institute of Standards and Technology, Boulder, Colorado 80305, USA \\ ${ }^{13}$ Service des Basses Températures, Commissariat à l'Energie Atomique, 38054 Grenoble, France \\ ${ }^{14}$ Department of Physics, University of Illinois at Urbana-Champaign, Urbana, Illinois 61801, USA \\ ${ }^{15}$ Department of Astronomy, University of Illinois at Urbana-Champaign, Urbana, Illinois 61801, USA \\ ${ }^{16}$ Department of Physics, Harvard University, Cambridge, Massachusetts 02138, USA \\ ${ }^{17}$ Kavli Institute for the Physics and Mathematics of the Universe (WPI), UTIAS, The University of Tokyo, \\ Kashiwa, Chiba 277-8583, Japan \\ ${ }^{18}$ Aix-Marseille Université, CNRS/IN2P3, CPPM, Marseille 13288, France \\ ${ }^{19}$ Physics Department, Brookhaven National Laboratory, Upton, New York 11973, USA \\ ${ }^{20}$ Department of Physics, Enrico Fermi Institute, University of Chicago, Chicago, Illinois 60637, USA
}

(Received 21 June 2021; accepted 12 August 2021; published 4 October 2021)

We present results from an analysis of all data taken by the BICEP2, Keck Array, and BICEP3 CMB polarization experiments up to and including the 2018 observing season. We add additional Keck Array observations at $220 \mathrm{GHz}$ and BICEP3 observations at $95 \mathrm{GHz}$ to the previous $95 / 150 / 220 \mathrm{GHz}$ dataset. The $Q / U$ maps now reach depths of 2.8, 2.8, and $8.8 \mu \mathrm{K}_{\mathrm{CMB}}$ arcmin at 95, 150, and $220 \mathrm{GHz}$, respectively, over an effective area of $\approx 600$ square degrees at $95 \mathrm{GHz}$ and $\approx 400$ square degrees at 150 and $220 \mathrm{GHz}$. The $220 \mathrm{GHz}$ maps now achieve a signal-to-noise ratio on polarized dust emission exceeding that of Planck at $353 \mathrm{GHz}$. We take auto- and cross-spectra between these maps and publicly available WMAP and Planck maps at frequencies from 23 to $353 \mathrm{GHz}$ and evaluate the joint likelihood of the spectra versus a multicomponent model of lensed $\Lambda \mathrm{CDM}+r+$ dust + synchrotron + noise. The foreground model has seven parameters, and no longer requires a prior on the frequency spectral index of the dust emission taken from measurements on other regions of the sky. This model is an adequate description of the data at the current noise levels. The likelihood analysis yields the constraint $r_{0.05}<0.036$ at $95 \%$ confidence. 
Running maximum likelihood search on simulations we obtain unbiased results and find that $\sigma(r)=0.009$.

These are the strongest constraints to date on primordial gravitational waves.

DOI: 10.1103/PhysRevLett.127.151301

Introduction.-The $\Lambda \mathrm{CDM}$ standard model of cosmology is able to describe the observable universe in a statistical manner using only six free parameters. Measurements of the cosmic microwave background (CMB) [1] are one the key pillars of this model and now constrain its parameters with percent-level precision (see most recently Ref. [2]).

The $\Lambda$ CDM model describes how the universe evolved from an initial high energy state $\left(T \gg 10^{12} \mathrm{~K}\right)$, and the conditions at that time can be inferred from observations: fractionally small, Gaussian, adiabatic perturbations with a slightly red power law spectrum $\left(n_{s} \lesssim 1\right)$. Inflationary theories naturally explain such conditions as the outcome of a prephase of exponential expansion during which the scale of the protouniverse increased by a factor of $\sim e^{60}$. Inflation makes an additional prediction which has not yet been observed-a background of tensor perturbations, also known as gravitational waves (see Ref. [3] for a review and citations to the original literature). There are many specific inflationary models and classes thereof. If we can detect or set limits on primordial gravitational waves we can set limits on these models [4], and probe physics at energy scales far higher than can ever be accessed in laboratory experiments.

A polarization pattern can be decomposed into $E$-mode (gradient) and $B$-mode (curl) components. Under the $\Lambda \mathrm{CDM}$ standard model the CMB polarization pattern is mostly $E$ mode, with a much smaller $B$-mode component which arises due to gravitational deflections (lensing) of the CMB photons after their last scattering [5]. Since primordial gravitational waves will produce $E$ modes and $B$ modes approximately equally it was realized in the late 1990s that the best way to search for them is to look for an excess $B$-mode signal [6-8]. Additional nonprimordial $B$ modes are produced by astrophysical foreground emissions, primarily from our own galaxy, but these have different frequency spectra than the $\mathrm{CMB}$ and can be separated from it using multifrequency measurements.

Our BICEP/Keck program first reported detection of an excess over the lensing $B$-mode expectation at $150 \mathrm{GHz}$ in Ref. [9]. In a joint analysis using multifrequency data from the Planck experiment it was shown that most or all of this is due to polarized emission from dust in our own galaxy ([10] hereafter BKP). In Ref. ([11] hereafter BK14) we improved the constraint using Keck Array data at $95 \mathrm{GHz}$ taken during the 2014 season, and in Ref. ([12] hereafter BK15) we improved again adding Keck Array data at 95 and $220 \mathrm{GHz}$ taken during the 2015 season. In this Letter [hereafter BK18] we add large amounts of new data taken by Keck Array at $220 \mathrm{GHz}$ and BICEP 3 at $95 \mathrm{GHz}$ during the 2016, 2017, and 2018 observing seasons. This paper follows BK15 very closely in the methods, structure, and, in places, even the wording, mainly just adding additional experimental data. This improves the constraint on primordial gravitational waves parametrized by the tensor-toscalar ratio $r$ by more than a factor of 2 over our previous result to $r_{0.05}<0.036$ at $95 \%$ confidence, setting important additional limits on inflationary models.

Instrument and observations.-The BICEP2 receiver observed at $150 \mathrm{GHz}$ from 2010-2012 [13]. The Keck Array was essentially five copies of BICEP2 running in parallel from 2012-2019, initially at $150 \mathrm{GHz}$ but switching over time to 95 and $220 \mathrm{GHz}$ [14]. BICEP3 is a single similar, but scaled up, receiver which commenced science observations in the 2016 Austral winter season [15]. Whereas the BICEP2 and Keck 150 and $220 \mathrm{GHz}$ receivers each contained $\approx 500$ bolometric detectors BICEP3 contains $\approx 2500$ detectors. The aperture size is also increased from $\approx 0.25 \mathrm{~m}$ to $\approx 0.5 \mathrm{~m}$. The Keck receivers were mounted on a single telescope mount (movable platform), while BICEP3 occupies a separate mount previously used for BICEP2 on a nearby building. All of these telescopes are located at the South Pole Station in Antarctica. The mounts scan the receivers across the sky, and the cryogenic detectors track the intensity of the incoming microwave radiation. The detectors are arranged as interleaved orthogonally polarized pairs in the focal planes and the pair difference timestreams are thus measures of the polarized emission from the sky [16]. At the South Pole the atmosphere is exceptionally transparent and stable at the observation frequencies ([17], Fig. 5).

BICEP2 and Keck Array both mapped a region of sky centered at RA $0 \mathrm{~h}$, Dec. $-57.5^{\circ}$ with an effective area of $\approx 400$ square degrees. BICEP3 has a larger instantaneous field of view and hence naturally maps a larger sky area with an effective area of $\approx 600$ square degrees. We have perturbed the center of the BICEP3 scan region such that most of this additional area falls on the higher declination side of the sky patch in an attempt to stay away from regions where the Planck data indicates polarized dust contamination may be higher. The BK15 dataset consisted of 4, 17, and 2 receiver years at 95, 150, and $220 \mathrm{GHz}$, respectively. BICEP3 is equivalent to about eight of the Keck Array $95 \mathrm{GHz}$ receivers [15] so the BK18 dataset is equivalent to about 28, 18, and 14 Keck receiver years at 95, 150, and $220 \mathrm{GHz}$, respectively.

Maps and power spectra.-We make maps and power spectra using the same procedures as in our previous series of papers. The timestream data are binned into pixels on the 

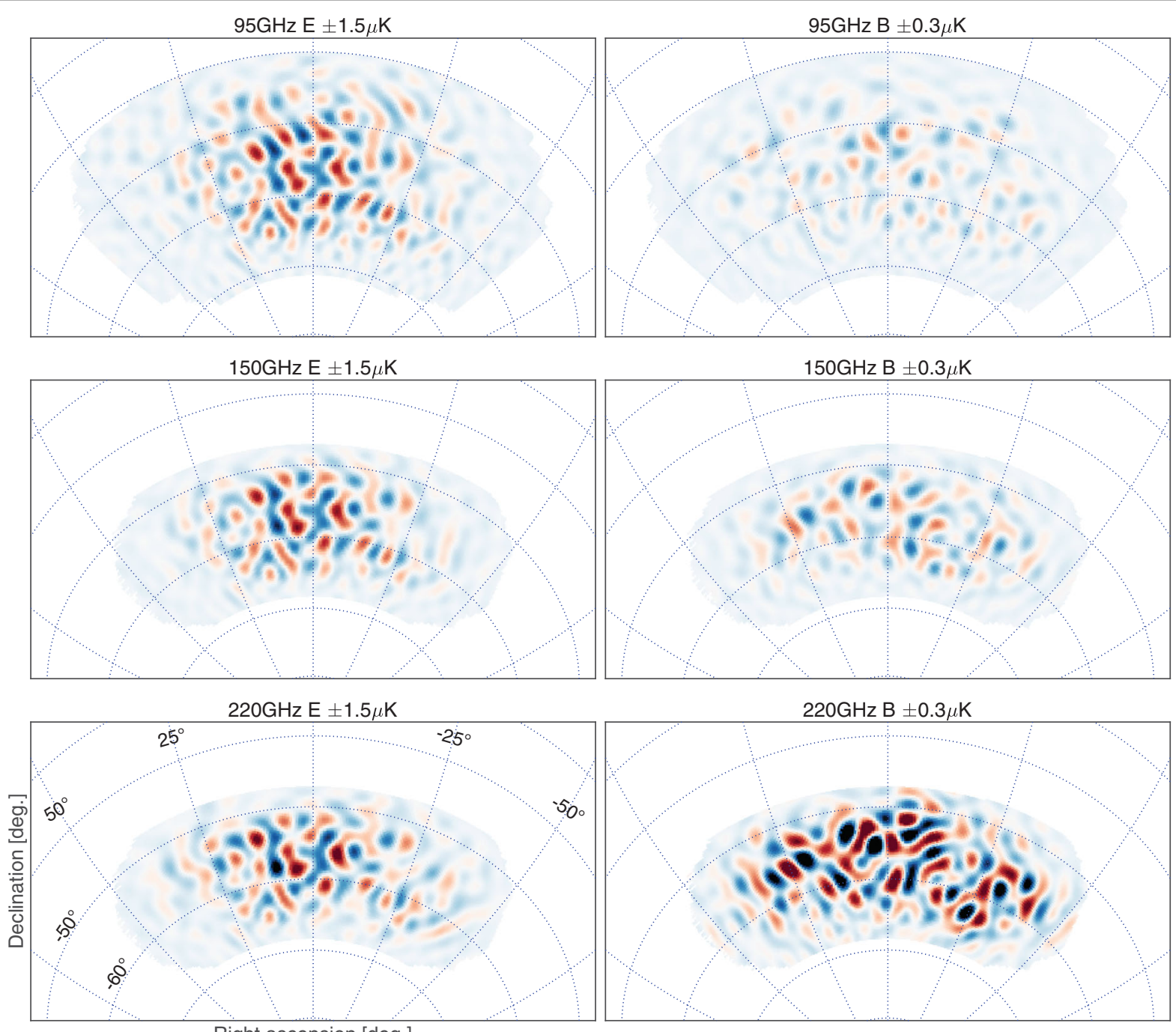

FIG. 1. $E$-mode (left column) and $B$-mode (right column) maps at 95, 150, and $220 \mathrm{GHz}$ in CMB units, and filtered to degree angular scales $(50<\ell<120)$. Note the differing color ranges left and right. The $E$ maps are dominated by $\Lambda$ CDM signal, and hence are highly correlated across all three bands. The $95 \mathrm{GHz} B$ map is approximately equal parts lensed- $\Lambda \mathrm{CDM}$ signal and noise. At $150 \mathrm{and} 220 \mathrm{GHz}$ the $B$ maps are dominated by polarized dust emission.

sky using knowledge of the pointing direction of the telescope at each moment in time, together with the relative angles from the telescope boresight to each individual detector pair. By taking data with the receivers rotated at a range of angles, maps of the Stokes parameters $Q$ and $U$ can be constructed.

The maps at each observing frequency are subjected to a matrix purification operation $[9,18]$ such that they contain only structures sourced by $B$ modes of the underlying sky pattern. This allows us to measure the $B$ modes in the presence of the much brighter $\Lambda \mathrm{CDM} E$ modes. The maps are then inverse noise variance apodized, Fourier transformed, and rotated from the $Q / U$ to the $E / B$ basis. In this paper we use our own maps at 95,150 , and $220 \mathrm{GHz}$ plus the 23 and $33 \mathrm{GHz}$ bands of WMAP [19,20] and the 30, 44, 143,217 , and $353 \mathrm{GHz}$ maps from the NPIPE processing of the Planck data $[21,22]$. For illustration purposes we can inverse Fourier transform to form $E / B$ maps. Figure 1 shows $E$ - and $B$-mode maps at 95,150 , and $220 \mathrm{GHz}$. (See Appendix A of the Supplemental Material for the full set of $T / Q / U$ maps [23]).

We take the variance within annuli of the Fourier plane to estimate the angular power spectra. Figure 2 shows the $E E$ and $B B$ auto- and cross-spectra for the BICEP/Keck bands plus the Planck $353 \mathrm{GHz}$ band which remains important for constraining the polarized dust contribution. Comparing this plot to Fig. 2 of BK15 we can see that the uncertainties are dramatically reduced for the auto- and cross-spectra of the 95 and $220 \mathrm{GHz}$ bands. The model plotted is a "baseline" lensed- $\Lambda \mathrm{CDM}+$ dust model from our previous BK15 analysis, which remains a good description of the data. The $E E$ spectra were not used to derive the model but 

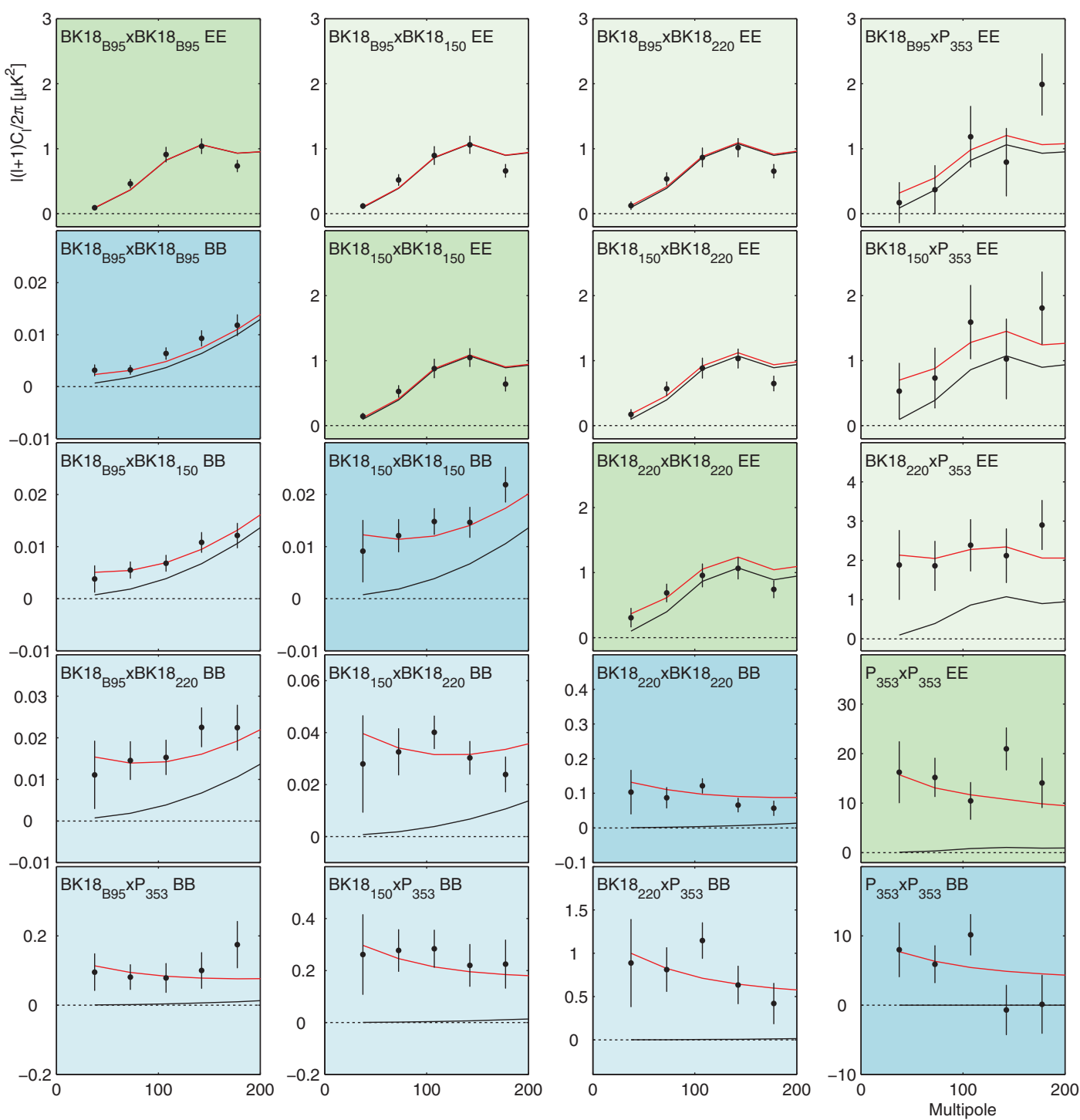

FIG. 2. $E E$ (green) and $B B$ (blue) auto- and cross-spectra calculated using the BICEP3 $95 \mathrm{GHz}$ map, the BICEP2/Keck $150 \mathrm{GHz}$ map, the Keck $220 \mathrm{GHz}$ map, and the Planck $353 \mathrm{GHz}$ map (with the auto-spectra in darker colors). The BICEP/Keck maps use all data taken up to and including the 2018 observing season-we refer to these as BK18. The black lines show the model expectation values for lensed $\Lambda \mathrm{CDM}$, while the red lines show the expectation values of a baseline lensed- $\Lambda \mathrm{CDM}+$ dust model from our previous BK15 analysis $\left(r=0, A_{d, 353}=4.7 \mu \mathrm{K}^{2}, \beta_{d}=1.6, \alpha_{d}=-0.4\right)$. Note that the model shown was fit to $B B$ only and did not use the BICEP3 $95 \mathrm{GHz}$ points shown (which are entirely new). The agreement with the spectra involving $95 \mathrm{GHz}$ and all the $E E$ spectra (under the assumption that $E E / B B=2$ for dust) is therefore a validation of the model.

agree well with it under the assumption that $E E / B B=2$ for dust, as is known to be close to the case $[37,38]$.

To test for systematic contamination we carry out our usual "jackknife" internal consistency (null) tests on the new 95 and $220 \mathrm{GHz}$ data as described in Appendixes B and $\mathrm{C}$ of the Supplemental Material [23]. Figure 3 (upper panel) shows the noise spectra for the three main BK18 bands after correction for the filter and beam suppression. In an auto-spectrum the quantity that determines the ability to constrain $r$ is the fluctuation of the noise band powers rather than their mean. The lower panel therefore shows the effective sky fraction as inferred from the fractional noise fluctuation. Together, these panels provide a useful synoptic measure of the loss of information due to noise, filtering, and $E E / B B$ separation in the lowest band powers (and we are glad to see taken up by others as, e.g., Fig. 6 of Ref. [39]).

Likelihood analysis.-We perform likelihood analysis using the methods introduced in BKP and refined in BK14 $\&$ BK15. We use the Hamimeche-Lewis approximation [40] to the joint likelihood of the ensemble of $66 \mathrm{BB}$ autoand cross-spectra taken between the BICEP/Keck, WMAP, and Planck maps. We compare the observed band power values for $20<\ell<330$ (nine band powers per spectrum) 


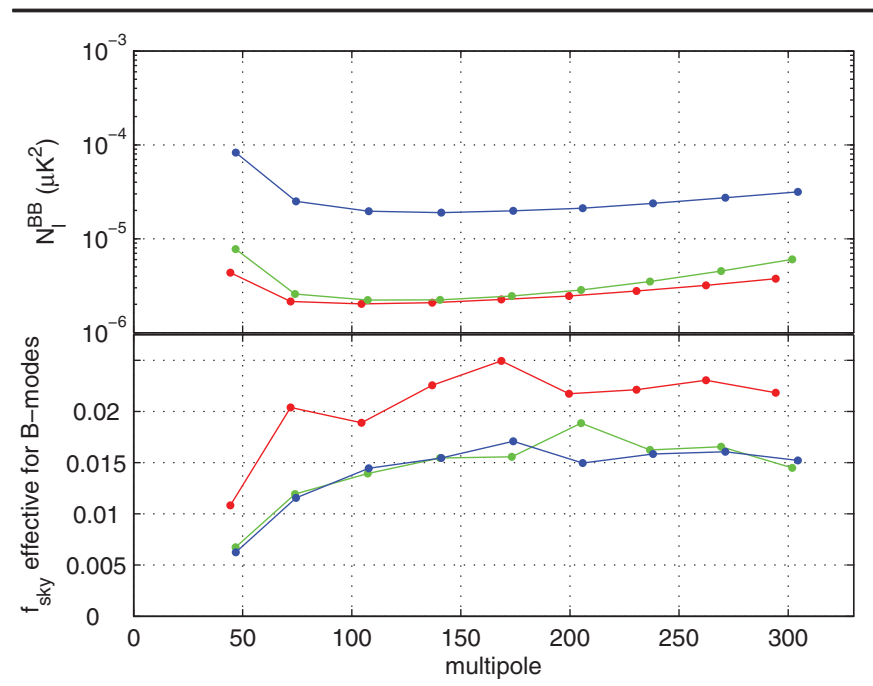

FIG. 3. Upper: the noise spectra of the BICEP3 $95 \mathrm{GHz}$ map (red), the BICEP2/Keck $150 \mathrm{GHz}$ map (green), and the Keck $220 \mathrm{GHz}$ maps (blue). The spectra are shown after correction for the filtering of signal which occurs due to the beam roll-off, timestream filtering, and $B$-mode purification. (Note that no $\ell^{2}$ scaling is applied.) Lower: the effective sky fraction as calculated from the ratio of the mean noise realization band powers to their fluctuation $f_{\text {sky }}(\ell)=(1 / 2 \ell \Delta \ell)\left\{\left[\sqrt{2} \overline{N_{b}}\right] /\left[\sigma\left(N_{b}\right)\right]\right\}^{2}$, i.e., the observed number of $B$-mode degrees of freedom divided by the nominal full-sky number. The turn down at low $\ell$ is due to mode loss to the timestream filtering and matrix purification.

to an eight parameter model of lensed $\Lambda \mathrm{CDM}+r+$ dust + synchrotron + noise and explore the parameter space using COSMOMC [41] (which implements a Markov chain Monte Carlo method). As in our previous analyses the band power covariance matrix is derived from 499 simulations of signal and noise, explicitly setting to zero terms such as the covariance of signal-only band powers with noise-only band powers or covariance of BICEP/Keck noise band powers with WMAP and Planck noise band powers (see Appendix H of BK15 and Appendix B of the Supplemental Material [42] for details). We deal with the differing sky coverage of the BICEP3 and BICEP2/Keck maps as described in Appendix D of the Supplemental Material [23]. The tensor-scalar power ratio $r$ is evaluated at a pivot scale of $0.05 \mathrm{Mpc}^{-1}$, and we fix the tensor spectral index $n_{t}=0$. A COSMOMC module containing the data and model is available for download at [43]. The following paragraphs briefly summarize the foreground model.

We include dust with amplitude $A_{d, 353}$ evaluated at $353 \mathrm{GHz}$ and $\ell=80$. The frequency spectral behavior is taken as a modified black body spectrum with $T_{d}=$ $19.6 \mathrm{~K}$ and frequency spectral index $\beta_{d}$. In a significant change from the baseline analysis choices of BK15, we remove the prior on the dust frequency spectral index which was previously applied based on Planck data in other regions of sky-with the improvement in the Keck
$220 \mathrm{GHz}$ sensitivity this prior is no longer needed. The spatial power spectrum is taken as a power law $\mathcal{D}_{\ell} \propto \ell^{\alpha_{d}}$ marginalizing uniformly over the (generous) range $-1<$ $\alpha_{d}<0$ [where $\mathcal{D}_{\ell} \equiv \ell(\ell+1) C_{\ell} / 2 \pi$ ]. Planck analysis consistently finds approximate power law behavior of both the $E E$ and $B B$ dust spectra with exponents $\approx-0.4[37,38]$.

We include synchrotron with amplitude $A_{\text {sync,23 }}$ evaluated at $23 \mathrm{GHz}$ (the lowest WMAP band) and $\ell=80$, assuming a simple power law for the frequency spectral behavior $A_{\text {sync }} \propto \nu^{\beta_{s}}$, and using a Gaussian prior $\beta_{s}=$ $-3.1 \pm 0.3$ taken from the analysis of WMAP 23 and $33 \mathrm{GHz}$ data in Ref [44]. We note that analysis of $2.3 \mathrm{GHz}$ data from S-PASS in conjunction with WMAP and Planck finds $\beta_{s}=-3.2$ with no detected trends with galactic latitude or angular scale [45], and that Ref. [46] analyzed the S-PASS and WMAP $23 \mathrm{GHz}$ data and found $\beta_{s}=$ $-3.22 \pm 0.06$ in the BICEP2 sky patch. The spatial power spectrum is taken as a power law $\mathcal{D}_{\ell} \propto \ell^{\alpha_{s}}$ marginalizing over the range $-1<\alpha_{s}<0$ [47]. Reference [45] finds a value at the bottom end of this range $(\approx-1)$ from the $S$ PASS data for $B B$ at high galactic latitude.

Finally we include sync-dust correlation parameter $\epsilon$ (called $\rho$ in some other papers $[38,45,48]$ ). As in BK15 we marginalize over the full possible range $-1<\epsilon<1$.

We hold the lensing $B$-mode spectrum fixed at that predicted for the Planck 2018 cosmological parameters ([2], Table II). Results of our baseline analysis are shown in Fig. 4 and yield the following statistics: $r_{0.05}=0.014_{-0.011}^{+0.010}$ ( $r_{0.05}<0.036$ at $95 \%$ confidence), $A_{d, 353}=4.4_{-0.7}^{+0.8} \mu \mathrm{K}^{2}$, $A_{\text {sync, } 23}<1.4 \mu \mathrm{K}^{2}$ at $95 \%$ confidence, and $\beta_{d}=1.49_{-0.12}^{+0.13}$. For $r$, the zero-to-peak likelihood ratio is 0.46. Taking $\frac{1}{2}\left[1-f\left(-2 \log L_{0} / L_{\text {peak }}\right)\right]$, where $f$ is the $\chi^{2} \mathrm{CDF}$ (for one degree of freedom), we estimate that the probability to get a likelihood ratio smaller than this is $11 \%$ if, in fact, $r=0$. As compared to the previous BK15 analysis, the likelihood curve for $r$ tightens considerably with the peak position shifting down slightly, and the $A_{d}$ curve tightens slightly. In addition the $A_{\text {sync }}$ curve now peaks at zero-the weak evidence for synchrotron we saw in BK15 is no longer present. (Using the S-PASS data [45] we estimate that the expectation is $A_{\text {sync, } 23} \approx 0.4 \mu \mathrm{K}^{2}$ in the BICEP/Keck field, which is consistent with our $A_{\text {sync }}$ likelihood curve.) In the BK15 analysis the constraint on $\beta_{d}$ was prior dominated, but for BK18 we see that the data is able to constrain this parameter almost as well as the prior previously did. Interestingly the peak value selected is very close to the mean value from Planck 2018 analysis of larger regions of sky $\beta_{d}=1.53$ [38].

The maximum likelihood model has parameters $r_{0.05}=0.011, \quad A_{d, 353}=4.4 \mu \mathrm{K}^{2}, \quad A_{\text {sync }, 23}=0.6 \mu \mathrm{K}^{2}$, $\beta_{d}=1.5, \quad \beta_{s}=-3.0, \quad \alpha_{d}=-0.66, \quad \alpha_{s}=0.00, \quad$ and $\epsilon=-0.11$. This model is an acceptable fit to the data with the probability to exceed (PTE) the observed value of 


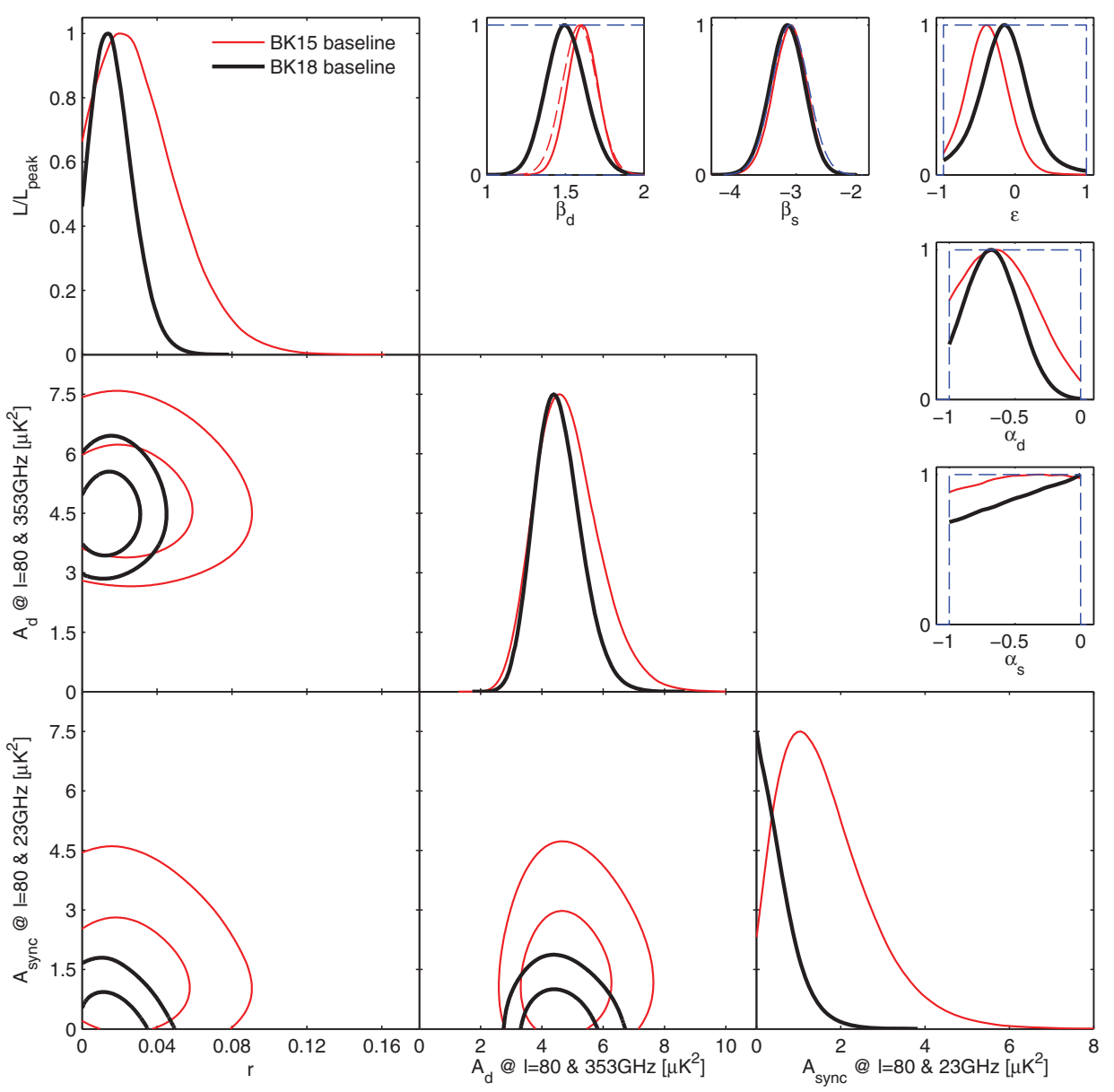

FIG. 4. Results of a multicomponent multispectral likelihood analysis of BICEP/Keck + WMAP, Planck data. The red faint curves are the baseline result from the previous BK15 paper (the black curves from Fig. 4 of that paper). The bold black curves are the new baseline BK18 results, adding a large amount of additional data at 95 and $220 \mathrm{GHz}$ taken by BICEP3 and Keck Array during the 20162018 observing seasons. The upper limit on the tensor-to-scalar ratio tightens to $r_{0.05}<0.036$ at $95 \%$ confidence. The parameters $A_{d}$ and $A_{\text {sync }}$ are the amplitudes of the dust and synchrotron $B$-mode power spectra, where $\beta$ and $\alpha$ are the respective frequency and spatial spectral indices. The correlation coefficient between the dust and synchrotron patterns is $\epsilon$. In the $\beta, \alpha$, and $\epsilon$ panels the dashed lines show the priors placed on these parameters (either Gaussian or uniform). Note that the Gaussian prior on $\beta_{d}$ has been removed going from BK15 to BK18.

$\chi^{2}$ being 0.94 . Thus, while the dust spectrum might in general be expected to exhibit fluctuations about power law spatial spectral behavior greater than that expected for a Gaussian random field, for the present the model continues to be an adequate description of the data-see Appendix D of the Supplemental Material [23] for further details.

In Appendix E of the Supplemental Material [23] we explore variation and validation of the likelihood. In Appendix E 2 of the Supplemental Material [23] we vary the baseline analysis choices and data selection, finding that these do not significantly alter the results, and that the data do not prefer allowing decorrelation of the dust pattern in the model. We also find that the value of $A_{d}$ is very similar when evaluated over the larger BICEP3 sky coverage region and the smaller BICEP2/Keck sky region. Freeing the amplitude of the lensing power we obtain
$A_{L}^{\mathrm{BB}}=1.03_{-0.09}^{+0.08}$, and the $r$ constraint hardly changes. In Appendix E 3 of the Supplemental Material [23] we verify that the likelihood analysis is unbiased, and in Appendix E 4 we explore a suite of alternate foreground models. As part of our standard data reduction we "deproject" leading order temperature to polarization leakage $[9,49]$-in Appendix F of the Supplemental Material [23] we quantify possible residual leakage and some other possible systematics.

Figure 5 shows the constraints in the $r$ vs $n_{s}$ plane for the Planck 2018 baseline analysis [2] and when adding in BK18 and BAO. The BK18 data shrink the contours in the vertical $(r)$ direction while the BAO data shrinks the contours in the horizontal $\left(n_{s}\right)$ direction and shifts the centroid slightly to the right. The $\phi^{2 / 3}$ model now lies outside the $95 \%$ contour as does the band of natural inflation models. 


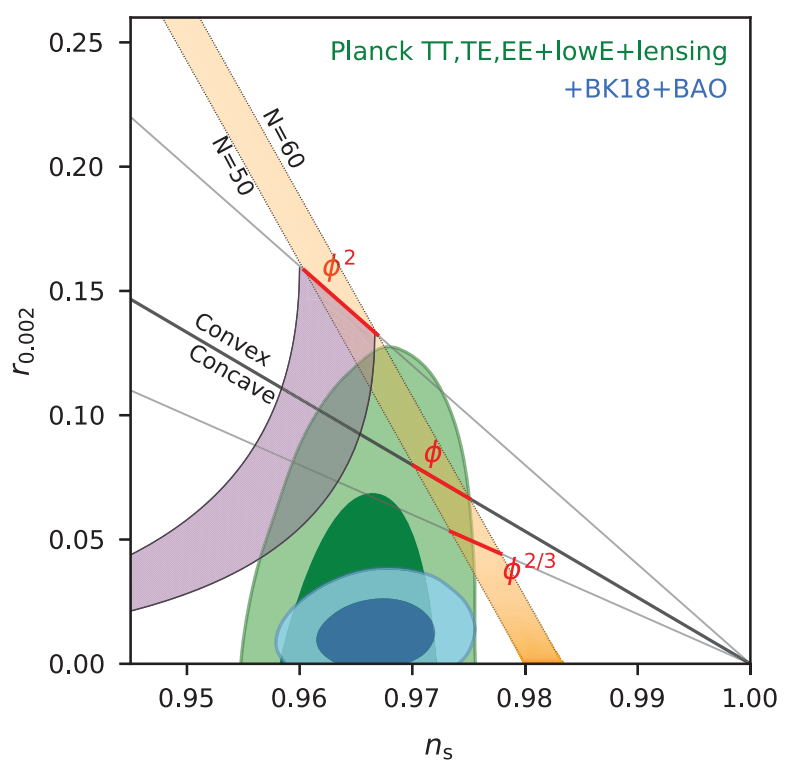

FIG. 5. Constraints in the $r$ vs $n_{s}$ plane for the Planck 2018 baseline analysis, and when also adding BICEP/Keck data through the end of the 2018 season plus BAO data to improve the constraint on $n_{s}$. The constraint on $r$ tightens from $r_{0.05}<$ 0.11 to $r_{0.05}<0.035$. This figure is adapted from Fig. 28 of Ref. [2] with the green contours being identical. Some additional inflationary models are added from Fig. 8 of Ref. [50] with the purple region being natural inflation.

Conclusions. - The BKP analysis yielded a 95\% confidence constraint $r_{0.05}<0.12$, which BK14 improved to $r_{0.05}<0.09$, and BK15 improved to $r_{0.05}<0.07$. The BK18 result described in this Letter, $r_{0.05}<0.036$, represents a fractional improvement equivalent to the two previous steps combined. The BK18 simulations have a median $95 \%$ upper limit of $r_{0.05}<0.019$.

The distributions of maximum likelihood $r$ values in simulations where the true value of $r$ is zero gave $\sigma\left(r_{0.05}\right)=$ 0.020 for BK15 which is reduced to $\sigma\left(r_{0.05}\right)=0.009$ for BK18 (see Appendix E 3 of the Supplemental Material [23] for details). Such simulations can also be used to investigate the degree to which the analysis is limited by foregrounds and lensing. Running the baseline BK18 analysis on simulations which contain no lensing $B$ modes gives $\sigma\left(r_{0.05}\right)=0.004$, while running without foreground parameters on simulations which contain no dust gives $\sigma\left(r_{0.05}\right)=0.007$. Running without foreground parameters on simulations which contain neither lensing or dust gives $\sigma\left(r_{0.05}\right)=0.002$.

Figure 6 shows the BK18 noise uncertainties in the $\ell \approx 80$ band powers as compared to the signal levels. The signal-to-noise ratio on polarized dust emission of our $220 \mathrm{GHz}$ band is now considerably higher than that of the Planck $353 \mathrm{GHz}$ band-i.e., the $220 \times 220$ noise point is much further below the dust band than the P353 $\times$ P353 point. Additional BICEP3 data taken during 2019-2021

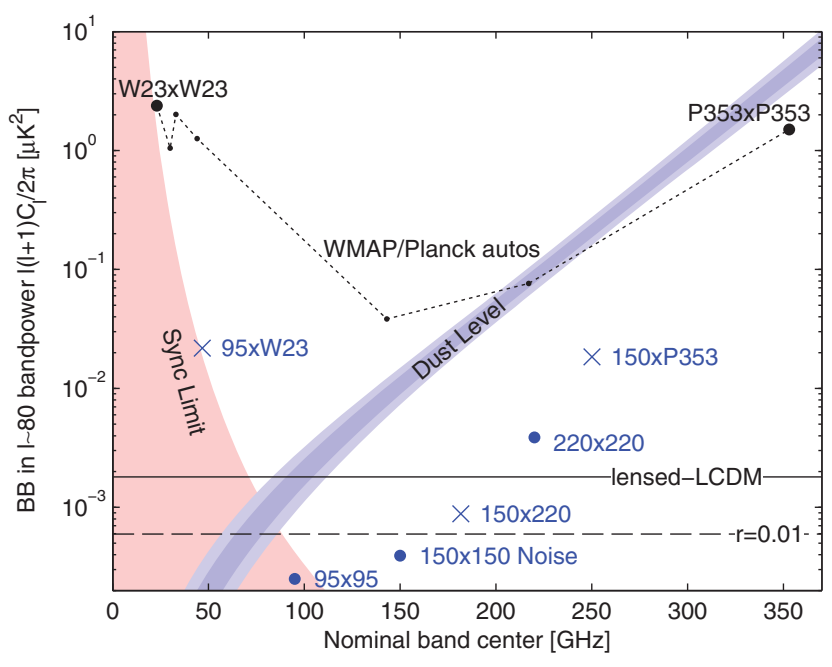

FIG. 6. Expectation values and noise uncertainties of the $\ell \sim 80$ $B B$ band power in the BICEP/Keck field. The solid and dashed black lines show the expected signal power of lensed $\Lambda \mathrm{CDM}$ and $r_{0.05}=0.01$. Since CMB units are used, the levels corresponding to these are flat with frequency. The blue bands show the 1 and $2 \sigma$ ranges of dust, and the red shaded region shows the 95\% upper limit on synchrotron in the baseline analysis including the uncertainties in the amplitude and frequency spectral index parameters $\left(A_{\mathrm{sync}, 23}, \beta_{s}\right.$ and $\left.A_{d, 353}, \beta_{d}\right)$. The BICEP/Keck autospectrum noise uncertainties are shown as large blue circles, and the noise uncertainties of the used WMAP and Planck singlefrequency spectra evaluated in the BICEP/Keck field are shown in black. The blue crosses show the noise uncertainty of selected cross-spectra, and are plotted at horizontal positions such that they can be compared vertically with the dust and sync curves.

will reduce the noise by a factor greater than 2 and $\sqrt{2}$ for $95 \times 95$ and $95 \times \mathrm{W} 23$, respectively, and we have also recorded additional data at 220 and $270 \mathrm{GHz}$.

Figure 7 shows the estimated CMB-only component of the BK18 $B$-mode band powers versus measurements from other experiments. See Appendix D of the Supplemental Material [23] for a description of how the CMB-only power spectrum estimate is calculated.

Figure 2 shows that the BK18 data are consistent with $\Lambda \mathrm{CDM}$ plus a remarkably simple dust only foreground model. Nevertheless as we move forward to even higher levels of sensitivity dust decorrelation, and foreground complexity more generally, will remain a serious concern. In addition, we are already in the regime where the sample variance of the lensing component dominates $\sigma(r)$. However, the lensing $B$ modes can be spatially separated from a primordial component and in this regard we have recently demonstrated a path forward by adding a "lensing template" derived from SPTpol and Planck data to the BK14 analysis, resulting in an improved constraint on $r$ [64].

The Keck Array mount has now been replaced by a larger, more capable machine and we are in the process of upgrading to a new system we call BICEP Array $[65,66]$. A 


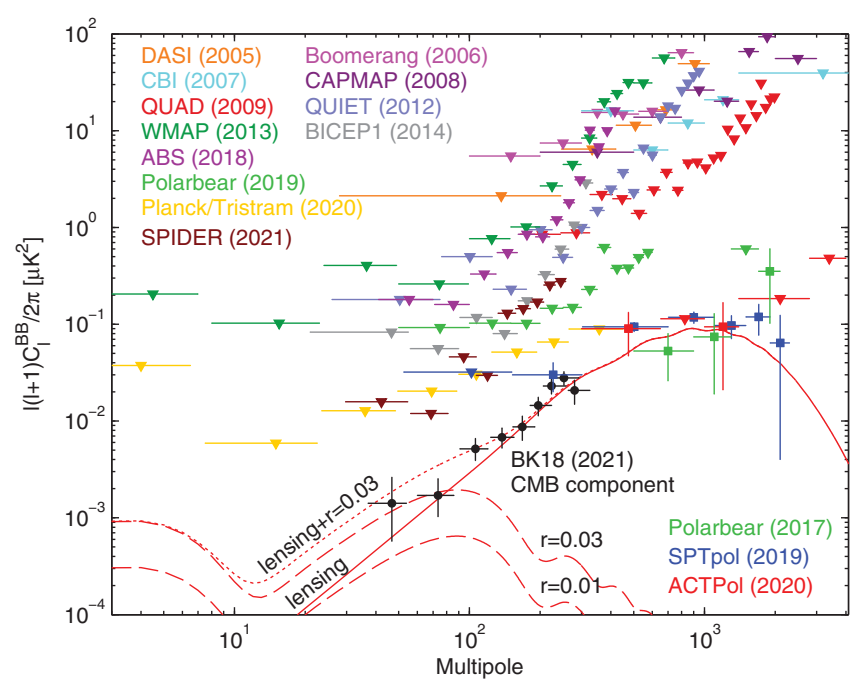

FIG. 7. Summary of CMB $B$-mode polarization upper limits [20,39,51-60] and detections [61-63]. Theoretical predictions are shown for the lensing $B$ modes (solid red) that peak at arcminute scales (multipole $\ell \sim 1000$ ), and for gravitational wave $B$ modes (dashed red) for two values of $r$ peaking at degree scales $(\ell \sim 80)$. The BK18 data are shown after removing Galactic foregrounds.

BICEP3 class receiver is now operating in the 30 or $40 \mathrm{GHz}$ band and in the coming years additional receivers will be installed at 95, 150, and 220 or $270 \mathrm{GHz}$. The system is projected to reach $\sigma(r) \sim 0.003$ within five years with delensing in conjunction with SPT3G.

The BICEP/Keck projects have been made possible through a series of grants from the National Science Foundation including Grants No. 0742818, No. 0742592, No. 1044978, No. 1110087, No. 1145172, No. 1145143 , No. 1145248 , No. 1639040, No. 1638957 , No. 1638978, and No. 1638970, and by the Keck Foundation. The development of antenna-coupled detector technology was supported by the JPL Research and Technology Development Fund, and by NASA Grants No. 06-ARPA206-0040, No. 10-SAT10-0017, No. 12SAT12-0031, No. 14-SAT14-0009, and No. 16-SAT-160002. The development and testing of focal planes were supported by the Gordon and Betty Moore Foundation at Caltech. Readout electronics were supported by a Canada Foundation for Innovation grant to UBC. Support for quasioptical filtering was provided by UK STFC Grant No. ST/N000706/1. The computations in this Letter were run on the Odyssey/Cannon cluster supported by the FAS Science Division Research Computing Group at Harvard University. The analysis effort at Stanford and S. L. A. C. is partially supported by the U.S. DOE Office of Science. We thank the staff of the U.S. Antarctic Program and in particular the South Pole Station without whose help this research would not have been possible. Most special thanks go to our heroic winter-overs Robert Schwarz, Steffen
Richter, Sam Harrison, Grantland Hall, and Hans Boenish. We thank all those who have contributed past efforts to the BICEP/Keck series of experiments, including the BICEP1 team. We also thank the Planck and WMAP teams for the use of their data, and are grateful to the Planck team for helpful discussions.

*pryke@physics.umn.edu

[1] A. A. Penzias and R. W. Wilson, A measurement of excess antenna temperature at $4080 \mathrm{Mc} / \mathrm{s}$, Astrophys. J. 142, 419 (1965).

[2] Planck Collaboration 2018 VI, Planck 2018 results VI. Cosmological parameters, Astron. Astrophys. 641, A6 (2020).

[3] M. Kamionkowski and E. D. Kovetz, The quest for B modes from inflationary gravitational waves, Annu. Rev. Astron. Astrophys. 54, 227 (2016).

[4] CMB-S4 Collaboration, CMB-S4 science book, first edition, arXiv: 1610.02743 .

[5] M. Zaldarriaga and U. Seljak, Gravitational lensing effect on cosmic microwave background polarization, Phys. Rev. D 58, 023003 (1998).

[6] U. Seljak, Measuring polarization in the cosmic microwave background, Astrophys. J. 482, 6 (1997).

[7] M. Kamionkowski, A. Kosowsky, and A. Stebbins, A Probe of Primordial Gravity Waves and Vorticity, Phys. Rev. Lett. 78, 2058 (1997).

[8] U. Seljak and M. Zaldarriaga, Signature of Gravity Waves in the Polarization of the Microwave Background, Phys. Rev. Lett. 78, 2054 (1997).

[9] BICEP2 Collaboration I, Detection of $B$-Mode Polarization at Degree Angular Scales by BICEP2, Phys. Rev. Lett. 112, 241101 (2014).

[10] BICEP2/Keck and Planck Collaborations, Joint Analysis of BICEP2/Keck Array and Planck Data, Phys. Rev. Lett. 114, 101301 (2015).

[11] Keck Array and BICEP2 Collaborations VI, Improved Constraints on Cosmology and Foregrounds from BICEP2 and Keck Array Cosmic Microwave Background Data with Inclusion of $95 \mathrm{GHz}$ Band, Phys. Rev. Lett. 116, 031302 (2016).

[12] Keck Array and BICEP2 Collaborations X, Constraints on Primordial Gravitational Waves Using Planck, WMAP, and New BICEP2/Keck Observations through the 2015 Season, Phys. Rev. Lett. 121, 221301 (2018).

[13] BICEP2 Collaboration II, BICEP2. II. Experiment and three-year data set, Astrophys. J. 792, 62 (2014).

[14] Keck Array and BICEP2 Collaborations V, BICEP2/KECK array V: Measurements of $B$-mode polarization at degree angular scales and $150 \mathrm{GHz}$ by the KECK array, Astrophys. J. 811, 126 (2015).

[15] BICEP/Keck Collaboration XV, BICEP/Keck XV: The BICEP3 CMB polarimeter and the first three year data set (to be published).

[16] BICEP2/Keck and Spider Collaborations, Antenna-coupled TES bolometers used in BICEP2, Keck Array, and spider, Astrophys. J. 812, 176 (2015). 
[17] C.-L. Kuo, Assessments of Ali, Dome A, and Summit camp for mm-wave observations using MERRA-2 reanalysis, Astrophys. J. 848, 64 (2017).

[18] Keck Array and BICEP2 Collaborations VII, BICEP2/ KECK array. VII. Matrix based E/B separation applied to BICEP2 and the KECK array, Astrophys. J. 825, 66 (2016).

[19] See http://lambda.gsfc.nasa.gov/product/map/dr5/m_products .cfm.

[20] C. L. Bennett, D. Larson, J. L. Weiland, N. Jarosik, G. Hinshaw, N. Odegard, K. M. Smith, R. S. Hill, B. Gold, M. Halpern, E. Komatsu, M. R. Nolta, L. Page, D. N. Spergel, E. Wollack, J. Dunkley, A. Kogut, M. Limon, S. S. Meyer, G. S. Tucker, and E. L. Wright, Nine-year Wilkinson Microwave Anisotropy Probe (WMAP) obsevations: Final maps and results, Astrophys. J. Suppl. Ser. 208, 20 (2013).

[21] See https://irsa.ipac.caltech.edu/data/Planck/release_3/ ancillary-data/HFI_Products.html.

[22] Planck Collaboration Int. LVII, Planck intermediate results LVII. Joint Planck LFI and HFI data processing, Astron. Astrophys. 643, A42 (2020).

[23] See Supplemental Material at http://link.aps.org/ supplemental/10.1103/PhysRevLett.127.151301 for appendixes, which include Refs. [24-36].

[24] Keck Array and BICEP2 Collaborations XI, BICEP2/Keck array XI: Beam characterization and temperature-to-polarization leakage in the BK15 data set, Astrophys. J. 884, 114 (2019).

[25] J. Willmert, Constraining inflationary B-modes with the BICEP/Keck array telescopes, Ph.D. thesis, University of Minnesota, 2019.

[26] Planck Collaboration Int. XXII, Planck intermediate results. XXII. Frequency dependence of thermal emission from Galactic dust in intensity and polarization, Astron. Astrophys. 576, A107 (2015).

[27] V. Pelgrims, S. E. Clark, B. S. Hensley, G. V. Panopoulou, V. Pavlidou, K. Tassis, H. K. Eriksen, and I. K. Wehus, Evidence for line-of-sight frequency decorrelation of polarized dust emission in Planck data, Astron. Astrophys. 647, A16 (2021).

[28] G. V. Panopoulou and D. Lenz, Maps of the number of $\mathrm{H} \mathrm{i}$ clouds along the line of sight at high galactic latitude, Astrophys. J. 902, 120 (2020).

[29] Planck Collaboration Int. L, Planck intermediate results L. Evidence of spatial variation of the polarized thermal dust spectral energy distribution and implications for CMB $B$ mode analysis, Astron. Astrophys. 599, A51 (2017).

[30] B. Thorne, J. Dunkley, D. Alonso, and S. Næss, The Python Sky Model: software for simulating the Galactic microwave sky, Mon. Not. R. Astron. Soc. 469, 2821 (2017).

[31] A. G. Kritsuk, S. D. Ustyugov, and M. L. Norman, The structure and statistics of interstellar turbulence, New J. Phys. 19, 065003 (2017).

[32] A. G. Kritsuk, R. Flauger, and S. D. Ustyugov, DustPolarization Maps for Local Interstellar Turbulence, Phys. Rev. Lett. 121, 021104 (2018).

[33] G. Martínez-Solaeche, A. Karakci, and J. Delabrouille, A 3D model of polarized dust emission in the Milky Way, Mon. Not. R. Astron. Soc. 476, 1310 (2018).

[34] F. Vansyngel, F. Boulanger, T. Ghosh, B. Wandelt, J. Aumont, A. Bracco, F. Levrier, P. G. Martin, and L. Montier,
Statistical simulations of the dust foreground to cosmic microwave background polarization, Astron. Astrophys. 603, A62 (2017).

[35] Keck Array and BICEP2 Collaborations IV, BICEP2/KECK $A R R A Y$. IV. Optical characterization and performance of the BICEP 2 and KECK ARRAY experiments, Astrophys. J. 806, 206 (2015).

[36] R. A. Battye, I. W. A. Browne, M. W. Peel, N. J. Jackson, and C. Dickinson, Statistical properties of polarized radio sources at high frequency and their impact on cosmic microwave background polarization measurements, Mon. Not. R. Astron. Soc. 413, 132 (2011).

[37] Planck Collaboration Int. XXX, Planck intermediate results XXX. The angular power spectrum of polarized dust emission at intermediate and high Galactic latitudes, Astron. Astrophys. 586, A133 (2016).

[38] Planck Collaboration 2018 XI, Planck 2018 results XI. Polarized dust foregrounds, Astron. Astrophys. 641, A11 (2020).

[39] POLARBEAR Collaboration, A measurement of the degreescale CMB $B$-mode angular power spectrum with POLARBEAR, Astrophys. J. 897, 55 (2020).

[40] S. Hamimeche and A. Lewis, Likelihood analysis of CMB temperature and polarization power spectra, Phys. Rev. D 77, 103013 (2008).

[41] A. Lewis and S. Bridle, Cosmological parameters from CMB and other data: A Monte Carlo approach, Phys. Rev. D 66, 103511 (2002).

[42] CMB-S4 Collaboration, CMB-S4: Forecasting constraints on primordial gravitational waves, arXiv:2008.12619 [Astrophys. J. (to be published)].

[43] http://bicepkeck.org.

[44] U. Fuskeland, I. K. Wehus, H. K. Eriksen, and S. K. Næss, Spatial variations in the spectral index of polarized synchrotron emission in the 9 yr WMAP sky maps, Astrophys. J. 790, 104 (2014).

[45] N. Krachmalnicoff, E. Carretti, C. Baccigalupi, G. Bernardi, S. Brown, B. M. Gaensler, M. Haverkorn, M. Kesteven, F. Perrotta, S. Poppi, and L. Staveley-Smith, S-PASS view of polarized Galactic synchrotron at $2.3 \mathrm{GHz}$ as a contaminant to $\mathrm{CMB}$ observations, Astron. Astrophys. 618, A166 (2018).

[46] U. Fuskeland, K. J. Andersen, R. Aurlien, R. Banerji, M. Brilenkov, H. K. Eriksen, M. Galloway, E. Gjerløw, S. K. Næss, T. L. Svalheim, and I. K. Wehus, Constraints on the spectral index of polarized synchrotron emission from WMAP and Faraday-corrected S-PASS data, Astron. Astrophys. 646, A69 (2021).

[47] J. Dunkley et al., Prospects for polarized foreground removal, AIP Conf. Proc. 1141, 222 (2009).

[48] S. K. Choi and L. A. Page, Polarized galactic synchrotron and dust emission and their correlation, J. Cosmol. Astropart. Phys. 12 (2015) 020.

[49] BICEP2 Collaboration III, BICEP2. III. Instrumental systematics, Astrophys. J. 814, 110 (2015).

[50] Planck Collaboration 2018 X, Planck 2018 results X. Constraints on inflation, Astron. Astrophys. 641, A10 (2020).

[51] E. M. Leitch, J. M. Kovac, N. W. Halverson, J. E. Carlstrom, C. Pryke, and M.W.E. Smith, Degree angular scale 
interferometer 3 year cosmic microwave background polarization results, Astrophys. J. 624, 10 (2005).

[52] T. E. Montroy et al., A measurement of the CMB $\langle\mathrm{EE}\rangle$ spectrum from the 2003 flight of BOOMERANG, Astrophys. J. 647, 813 (2006).

[53] J. L. Sievers, C. Achermann, J. R. Bond, L. Bronfman, R. Bustos, C. R. Contaldi, C. Dickinson, P. G. Ferreira, M. E. Jones, A. M. Lewis, B. S. Mason, J. May, S. T. Myers, N. Oyarce, S. Padin, T. J. Pearson, M. Pospieszalski, A. C. S. Readhead, R. Reeves, A. C. Taylor, and S. Torres, Implications of the cosmic background imager polarization data, Astrophys. J. 660, 976 (2007).

[54] CAPMAP Collaboration, New measurements of fine-scale CMB polarization power spectra from CAPMAP at both 40 and $90 \mathrm{GHz}$, Astrophys. J. 684, 771 (2008).

[55] QUaD Collaboration, Improved measurements of the temperature and polarization of the cosmic microwave background from QUaD, Astrophys. J. 705, 978 (2009).

[56] QUIET Collaboration, Second season quiet observations: Measurements of the cosmic microwave background polarization power spectrum at $95 \mathrm{GHz}$, Astrophys. J. 760, 145 (2012).

[57] BICEP1 Collaboration, Degree-scale cosmic microwave background polarization measurements from three years of BICEP1 data, Astrophys. J. 783, 67 (2014).

[58] A. Kusaka et al., Results from the Atacama B-mode Search (ABS) experiment, J. Cosmol. Astropart. Phys. 09 (2018) 005.

[59] M. Tristram, A. J. Banday, K. M. Górski, R. Keskitalo, C. R. Lawrence, K. J. Andersen, R. B. Barreiro, J.
Borrill, H. K. Eriksen, R. Fernandez-Cobos, T. S. Kisner, E. Martínez-González, B. Partridge, D. Scott, T. L. Svalheim, H. Thommesen, and I. K. Wehus, Planck constraints on the tensor-to-scalar ratio, Astron. Astrophys. 647, A128 (2021).

[60] SPIDER Collaboration, A constraint on primordial $B$-modes from the first flight of the SPIDER balloon-borne telescope, arXiv:2103.13334 [Astrophys. J. (to be published)].

[61] J. T. Sayre et al., Measurements of $B$-mode polarization of the cosmic microwave background from 500 square degrees of SPTpol data, Phys. Rev. D 101, 122003 (2020).

[62] S. K. Choi et al., The atacama cosmology telescope: a measurement of the cosmic microwave background power spectra at 98 and $150 \mathrm{GHz}, \mathrm{J}$. Cosmol. Astropart. Phys. 12 (2020) 045.

[63] POLARBEAR Collaboration, A measurement of the cosmic microwave background $B$-mode polarization power spectrum at subdegree scales from two years of POLARBEAR data, Astrophys. J. 848, 121 (2017).

[64] BICEP2/Keck and SPTpol Collaborations, A demonstration of improved constraints on primordial gravitational waves with delensing, Phys. Rev. D 103, 022004 (2021).

[65] H. Hui and the BICEP/Keck Collaboration, BICEP Array: a multi-frequency degree-scale CMB polarimeter, Proc. SPIE Int. Soc. Opt. Eng. 10708, 1070807 (2018).

[66] L. Moncelsi and the BICEP/Keck Collaboration, Receiver development for BICEP Array, a next-generation CMB polarimeter at the South Pole, Proc. SPIE Int. Soc. Opt. Eng. 11453, 1145314 (2020). 